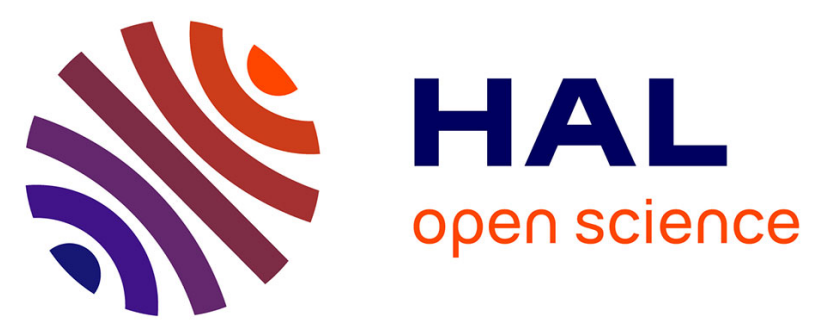

\title{
Evaluation of the noise properties of a dual-frequency VECSEL for compact Cs atomic clocks (Poster)
}

\author{
Paul Dumont, Jean-Marie Danet, D Holleville, S Guerandel, G Baili, L \\ Morvan, G Pillet, D Dolfi, I Gozhyk, G Beaudoin, et al.
}

\section{- To cite this version:}

Paul Dumont, Jean-Marie Danet, D Holleville, S Guerandel, G Baili, et al.. Evaluation of the noise properties of a dual-frequency VECSEL for compact Cs atomic clocks (Poster). Photonics West LASE, Conference on Vertical External Cavity Surface Emitting Lasers (VECSELs) V , Feb 2015, San Francisco, United States. pp.93490S, 10.1117/12.2077663 . hal-01171371

\section{HAL Id: hal-01171371}

https://hal-iogs.archives-ouvertes.fr/hal-01171371

Submitted on 3 Jul 2015

HAL is a multi-disciplinary open access archive for the deposit and dissemination of scientific research documents, whether they are published or not. The documents may come from teaching and research institutions in France or abroad, or from public or private research centers.
L'archive ouverte pluridisciplinaire HAL, est destinée au dépôt et à la diffusion de documents scientifiques de niveau recherche, publiés ou non, émanant des établissements d'enseignement et de recherche français ou étrangers, des laboratoires publics ou privés. 


\title{
Evaluation of the noise properties of a dual-frequency VECSEL for compact Cs atomic clocks
}

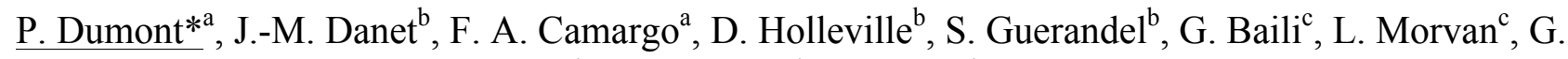

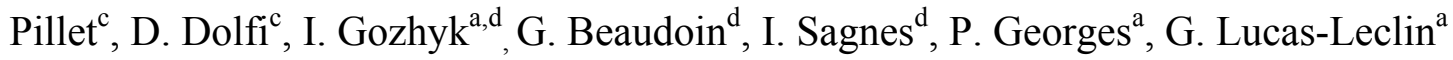 \\ ${ }^{a}$ Laboratoire Charles Fabry, Institut d'Optique, CNRS, Univ Paris-Sud 11, Palaiseau, France ; \\ ${ }^{\mathrm{b}}$ LNE-SYRTE, Observatoire de Paris, CNRS, UPMC, Paris, France ; \\ ${ }^{\mathrm{c}}$ Thales Research \& Technology, Palaiseau, France ; \\ ${ }^{\mathrm{d}}$ Laboratoire de Photonique et de Nanostructures, CNRS UPR20, Marcoussis, France \\ *paul.dumont@institutoptique.fr
}

\begin{abstract}
We evaluate a dual-frequency and dual-polarization optically-pumped semiconductor laser emitting at $852 \mathrm{~nm}$ as a new laser source for compact atomic clocks based on the coherent population trapping (CPT) technique. The frequency difference between the laser modes is tunable to $9.2 \mathrm{GHz}$ corresponding to the ground state hyperfine-split of Cs. Impact of the laser noise has been investigated. Laser relative intensity noise is limited by the pump-RIN transfer to a level of $110 \mathrm{~dB} / \mathrm{Hz}$. Laser frequency noise shows excess mechanical and technical noise resulting in a laser linewidth of $1 \mathrm{MHz}$ at $1 \mathrm{~s}$ in lock operation. The noise performance and spectral properties of the laser are already adequate to realize CPT experiments and should result in Allan standard-deviation of the clock below $1 \times 10^{-12}$ at 1 second.
\end{abstract}

Keywords: Dual-frequency laser, Vertical External Cavity Surface-Emitting Lasers, Atomic Clocks, Laser Noise.

\section{INTRODUCTION}

Atomic frequency references provide high-precision stable signals, which are crucial in the most demanding applications as satellite positioning, high bitrate communication networks, or high-end inertial navigation. Because of their potential for size reduction, atomic clocks based on the coherent population trapping (CPT) of ${ }^{133} \mathrm{Cs}$ atoms have raised a considerable interest within the last decade in the scientific and industrial community [1], [2]. In a CPT-based Cs atomic clock, the microwave interrogation at $9.192 \mathrm{GHz}$ is optically-induced by two phase-coherent laser fields at resonance with a common excited state. When this resonance is reached, the atoms are trapped in a so-called dark state, resulting in a narrow transmission line in the atomic absorption spectrum. Among the different optical configurations which influence the CPT signal, those combining cross-polarized excitation scheme and temporal Ramsey-like pulsed interrogation have demonstrated highly contrasted and narrow-linewidth CPT resonance [3]. Such configuration was recently proven to reach short-term frequency stability of a few $10^{-13}$ at one second [4].

As miniaturization is a challenge for CPT clocks, a simplified optical bench using a single laser source would be a serious improvement. We propose here a new laser architecture for such CPT clocks, consisting in a dual-frequency and dual-polarization laser emitting simultaneously two cross-polarized longitudinal modes inside the same laser cavity [5]. As the noise properties of optically carried microwave signal impact the clock signal, we describe the thorough evaluation of the intensity and frequency noise of our laser.

\section{DESCRIPTION OF THE LASER SOURCE}

The laser architecture is a Vertical External Cavity Semiconductor Laser (VECSEL), also known as a semiconductor disk laser (SDL). It consists in a semiconductor gain medium grown onto a Bragg mirror, pumped by a high-power fibercoupled laser diode at $670 \mathrm{~nm}$. The semiconductor chip is designed to ensure efficient pump absorption and light emission at wavelengths close to $852 \mathrm{~nm}$, corresponding to the $\mathrm{D}_{2}$ line of ${ }^{133} \mathrm{Cs}[6]$. 


\subsection{Semiconductor structure}

The semiconductor chip is a conventional multilayer active structure, grown with metal-organic chemical-vapor deposition on a $350 \mu$ m-thick GaAs substrate. A distributed Bragg reflector (DBR), composed of 32.5 pairs of $\lambda / 4$-thick $\mathrm{AlAs} / \mathrm{Al}_{0.225} \mathrm{Ga}_{0.775} \mathrm{As}$ layers, provides a $80-\mathrm{nm}$ large high reflectivity centered at $850 \mathrm{~nm}$, with $R>99.94 \%$ at $852 \mathrm{~nm}$. The $30 \lambda / 4$-thick active structure is composed of seven $8 \mathrm{~nm}$-thick GaAs quantum wells (QW) embedded in pump absorbing $\mathrm{Al}_{0.225} \mathrm{Ga}_{0.775} \mathrm{As}$ barriers; the $\mathrm{QW}$ are positioned at the antinodes of the optical standing wave following a 11110101001000 sequence (starting at the air surface, see Figure 1) resulting in almost identical pumping for each QW. The pump absorption is $90 \%$ of the internal pump power in a single pass. Two 30 -nm thick $\mathrm{Al}_{0.39} \mathrm{Ga}_{0.61} \mathrm{As}$ layers form potential barriers on each side of the active cavity, for carrier confinement. Finally, a $50 \mathrm{~nm}$-thick InGaP capping layer protects the cavity from Al-oxidation.

The theoretical design of the structure has been adjusted by comparison with measurements of its reflectivity spectrum (with and without an anti-reflection coating). We have deduced the actual resonant factor $\Gamma(\lambda)$ of the gain, which includes the enhancement of the optical field by the micro-cavity and the effect of the periodic gain [7] (Fig 1). We predict a contribution of the microcavity of 3 and a value $\Gamma=6.5$ at $852 \mathrm{~nm}$ at the temperature of $22^{\circ} \mathrm{C}$. The detuning between the QW absorption and the microcavity resonance is $12 \mathrm{~nm}$, close to optimal for our operating conditions.

Finally, the modal gain of the active structure has been measured as a function of the pump intensity in a dedicated setup (Fig. 2). The intracavity losses are varied by changing the output coupler transmission and adjusting the orientation of an intracavity uncoated glass plate. In order to suppress the pump-induced heating of the structure, the pump laser diode was first operating in QCW with a repetition rate of $33 \mathrm{~Hz}$ and a pulse duration of $90 \mu$ s (corresponding to a duty cycle of $0.3 \%$ ). Under these pumping conditions, the optical gain reaches $12 \%$; but the maximum achievable gain falls to $8 \%$ under $\mathrm{CW}$ pumping. We have compared these results with an analytical expression of the modal gain, taking into account the thermally-induced spectral shifts of the micro-cavity resonance $(0.06 \mathrm{~nm} / \mathrm{K})$ and of the gain $(0.32 \mathrm{~nm} / \mathrm{K})-\mathrm{as}$ deduced from the characterization of the structure - and the exponential decrease of the gain with a characteristic temperature $\mathrm{T}_{0}=70 \mathrm{~K}$. A thermal resistance of $60 \mathrm{~K} / \mathrm{W}$ with regard to the pump power has been considered. From these preliminary characterizations of the active structure, it appears that its design will provide a high gain at $852 \mathrm{~nm}$, without further thermal management post-processing.
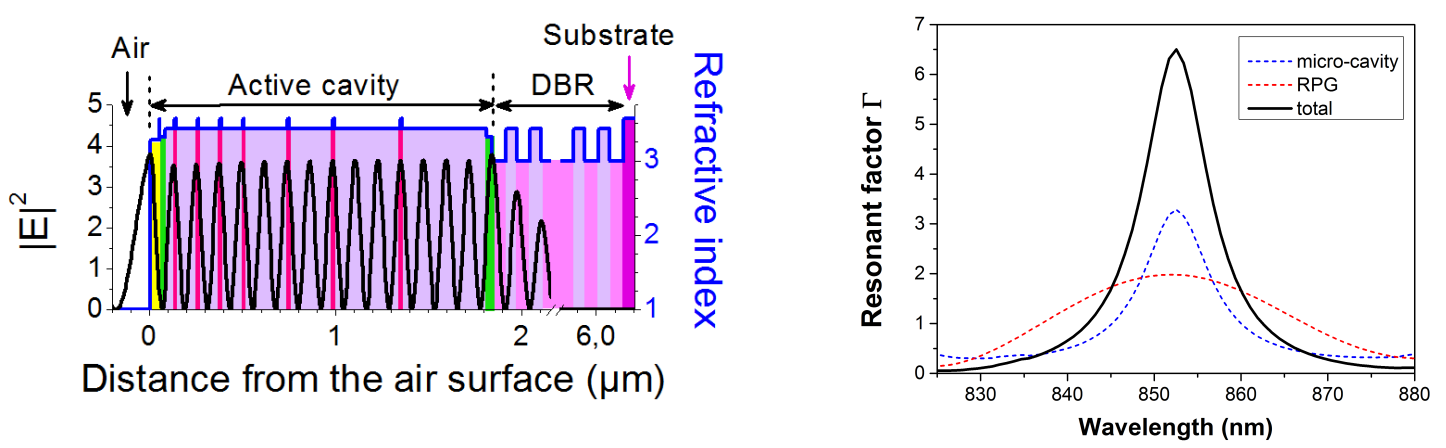

Figure 1: (left) Semiconductor structure design and optical field. (right) Resonant factor computed from reflectivity measurements at $\mathrm{T}=22^{\circ} \mathrm{C}$ with contributions of the resonant micro-cavity and the resonant periodic gain (RPG). 


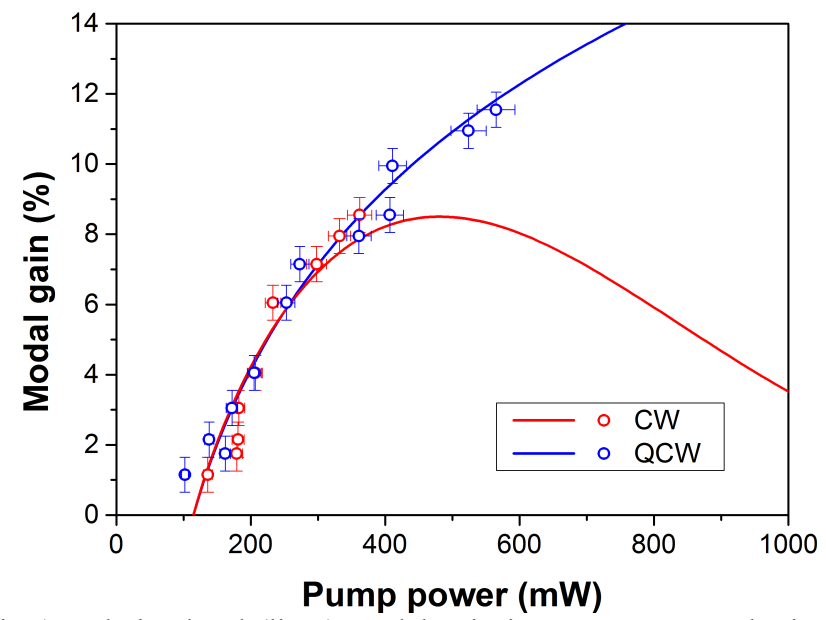

Figure 2: Experimental (points) and simulated (lines) modal gain in percent versus the incident pump power. Heatsink temperature is $15^{\circ} \mathrm{C}$. Laser spot is $84 \mu \mathrm{m} \times 101 \mu \mathrm{m}$.

\subsection{Laser description and free-running operation}

The laser cavity is linear, composed by the active structure and closed by a low-transmission ( $\mathrm{T}=0.5 \%)$ concave output coupler with a radius of curvature of $15 \mathrm{~mm}$ (Fig. 3). The laser emission properties are controlled by adding freespace intracavity elements: a $50 \mu \mathrm{m}$-thick Fabry-Perot etalon forces the single-mode emission at the desired wavelength; the fine tuning of the laser wavelength is obtained using a piezo-transducer (PZT) glued on the output coupler. A birefringent $\mathrm{YVO}_{4}$ plate induces a lateral separation of $50 \mu \mathrm{m}$ in the gain medium between the ordinary and extraordinary polarized beams, enough to lift the degeneracy between polarizations; a MgO-SLT electro-optical crystal allows the fine adjustment, through temperature and voltage, of the frequency difference $\Delta v$ between the two crosspolarized lines. With all the intra-cavity elements, the free spectral range of the laser cavity is $12 \mathrm{GHz}$, higher than the targeted frequency difference $v_{C S}=9.192 \mathrm{GHz}$, and corresponding to an optical cavity length $L_{c a v}=12.5 \mathrm{~mm}$. The pump optics, the semiconductor chip and the intra-cavity elements are integrated in a compact $(90 \mathrm{~mm} \times 90 \mathrm{~mm} \times 40 \mathrm{~mm})$ thermo-regulated casing.

The laser threshold is reached for an incident pump power of $0.35 \mathrm{~W}$ focused with a $50^{\circ}$ angle on $76 \mu \mathrm{m} \times 118 \mu \mathrm{m}$ spot. At the maximum pump power of $1 \mathrm{~W}$ and for a laser wavelength of $852 \mathrm{~nm}$, the optical power is $8 \mathrm{~mW}$ on each cross-polarized laser line. The frequency difference $\Delta v$ between the two laser frequencies is tunable with the electrooptic temperature $(1.4 \mathrm{GHz} / \mathrm{K})$ and the voltage $(1.6 \mathrm{MHz} / \mathrm{V})$; this allows the fine tuning of $\Delta v$ at the Cs reference frequency at $9.192 \mathrm{GHz}$.

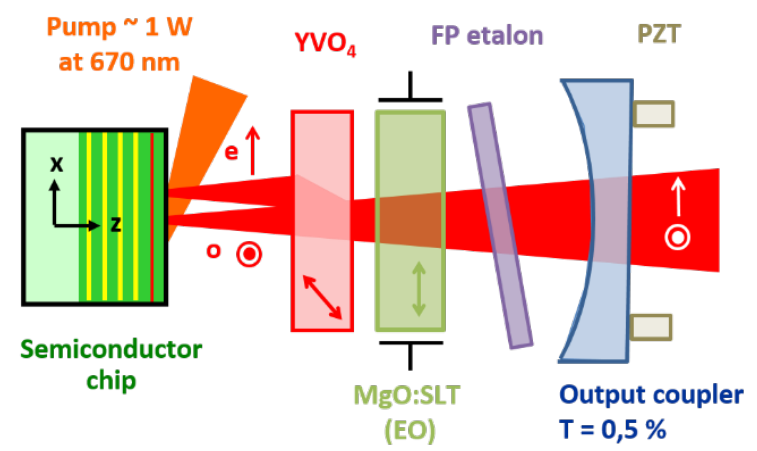

Figure 3: Laser cavity set-up. PZT: piezo-transducer; FP etalon: Fabry-Perot etalon; EO: Electro-optic plate.

\subsection{Laser stabilization set-up and locked operation}

The two cross-polarized laser lines can be tuned to the two targeted Cs hyperfine transitions distant from $9.192 \mathrm{GHz}$ : on one hand, the ordinary-polarized laser frequency is locked onto a Doppler-free transition in a Cs cell at room 
temperature, through feedback to the piezo-electric transducer glued to the output coupler and to the pump diode current. The error signal of this servo loop is generated using a pump-probe saturated absorption set-up where the pump beam is modulated at $100 \mathrm{kHz}$ to realize heterodyne detection on the probe signal. On the other hand, the frequency difference between laser modes can be locked onto a RF local oscillator using an optical-phase lock loop on the E.O. voltage, as described in [8], but it has not been used in the following experiments.

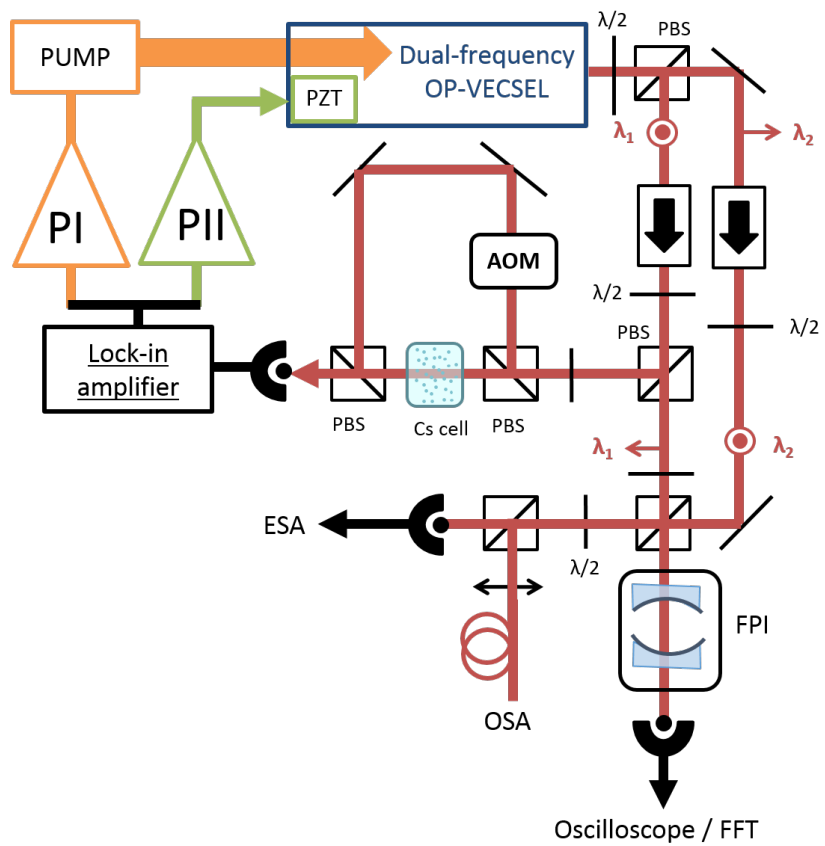

Figure 4: Laser stabilization experimental set-up. PBS: Polarizer Beam Splitter; $\lambda / 2$ : Half wave plate; AOM: Acoustooptic modulator; FPI: Fabry-Perot Interferometer; OSA: Optical spectrum analyzer; ESA: Electrical spectrum analyzer; PI: Proportional integrator servo; PII: Proportional with a 2-integration-stage servo; FFT : Fast Fourier Transform spectrum analyzer.

\section{ANALYSIS OF THE LASER INTENSITY NOISE}

The noise properties of the stabilized laser emission are investigated in order to evaluate the contribution of the dualfrequency VECSEL to the performance of a CPT atomic clock [9]. In this section we study the laser intensity fluctuations for the free running laser, as it directly impacts the CPT signal through amplitude noise on the optically generated microwave signal.

The power fluctuations of the whole laser output and of each linearly-polarized laser line are measured simultaneously with identical $1 \mathrm{GHz}$-bandwidth photodetectors. The signals are recorded with a digital oscilloscope (sampling rate up to $20 \mathrm{GSa} / \mathrm{s}$, record length up to 10,000,000 points, Agilent Infiniium). From the time evolution of the signals, we compute the relative intensity noise $(R I N)$ spectra through FFT algorithm. The measurements of the RIN show an identical flat noise level for each cross-polarized mode around $-110 \mathrm{~dB} / \mathrm{Hz}$, between $2 \mathrm{kHz}$ to $300 \mathrm{kHz}$ (Fig. 4), originating from direct conversion of pump RIN through carrier fluctuations [10]. We can notice technical noise peaks around $40-50 \mathrm{kHz}$ coming from pump driver. For frequencies above $300 \mathrm{kHz}$, the intensity noise decreases, revealing the laser cut-off frequency around $2 \mathrm{MHz}$. Thanks to the class-A dynamical behavior of the OP-VECSEL, the RIN spectra do not exhibit a relaxation oscillation peak at this frequency. The peak around $53 \mathrm{MHz}$ is also observed in the pump RIN spectrum. Above $70 \mathrm{MHz}$ the $R I N$ reaches the oscilloscope noise floor at a level around $-145 \mathrm{~dB} / \mathrm{Hz}$. 


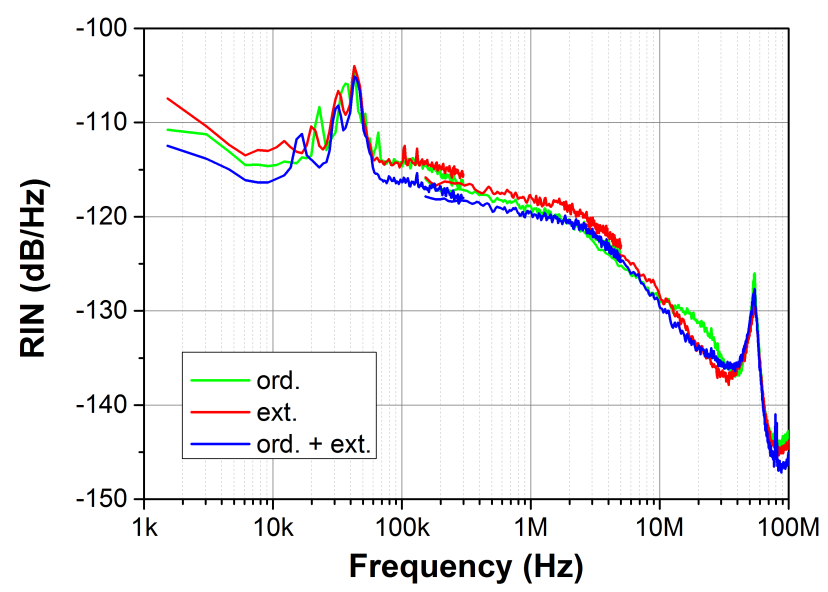

Figure 4: Experimental laser $R I N$ for ordinary (green) and extraordinary (red) polarized modes and for superposition of both modes (blue).

From the simultaneous oscilloscope records of the power fluctuations of the two cross-polarized laser lines, we compute the correlation spectrum between their intensity noises defined as [11]:

$$
\Theta(f)=\frac{\left\langle\delta \widetilde{V}_{1}(f) \delta \widetilde{V}_{2}^{*}(f)\right\rangle}{\sqrt{\left\langle\left|\delta \widetilde{V}_{1}\right|^{2}\right\rangle\left\langle\left|\delta \widetilde{V}_{2}\right|\right\rangle^{2}}}
$$

Where $V_{1}$ et $V_{2}$ are respectively the signals from the two photodiodes. The correlation function takes complex values and is described by its modulus $|\Theta(f)|$, the correlation amplitude, and by its phase given by $\Phi=\operatorname{Arg}(\Theta)$. This function verifies $|\Theta(f)|<1,|\Theta(f)|=1$ being a perfect correlation at frequency $\mathrm{f}$. The phase correlation indicates the operating point of the dual-frequency laser, which is either in-phase, $\operatorname{Arg}(\Theta(\mathrm{f}))=0$, or anti-phase $\operatorname{Arg}(\Theta)=\pi$. The experimental results are shown in figure 5 . As we can see, the modulus of the correlation function is above $-5 \mathrm{~dB}$ from $80 \mathrm{kHz}$ to 70 $\mathrm{MHz}$ except for a dip at $1.4 \mathrm{MHz}$ which is associated with a switch in the laser operation, from anti-phase to in-phase oscillations of the two modes. The cut-off in the correlation modulus above $70 \mathrm{MHz}$ is due to the decrease of both $R I N$ below the technical noise floor. Those results are consistent with other measurements undertaken with OP-VECSEL at $\lambda$ $=1 \mu \mathrm{m}$ for intermediate and strong coupling between the two laser modes as reported in [11], and is analog to the behavior of a two-coupled-mechanical-oscillator system. For frequencies in the $80 \mathrm{kHz}-1 \mathrm{MHz}$ range, the power fluctuations of the two cross-polarized lines are anti-correlated as a result of their strong coupling. Above $1 \mathrm{MHz}$, inphase fluctuations of the two lines dominate, which we interpret as a decrease of photon coupling in this frequency range: both modes operate as two independent lasers pumped by the same source. The correlation between the two lines is also evidenced in the RIN measurement of the total power (Fig. 4), where we observe a decrease of $3 \mathrm{~dB}$ at $100 \mathrm{kHz}$. In fact, assuming the $R I N$ and the power of each mode are identical, the output laser $R I N_{1+2}$ can be written as:

$$
R I N_{1+2}=\frac{R I N_{1,2}}{2}\{1+|\Theta(f)| \cos (\operatorname{Arg}[\Theta(f)])\}
$$

In our conditions, it could result in a $5 \mathrm{~dB}$ decrease at $100 \mathrm{kHz}$ of the $R I N$ according to the experimental correlation spectrum $\Theta(f)$. 


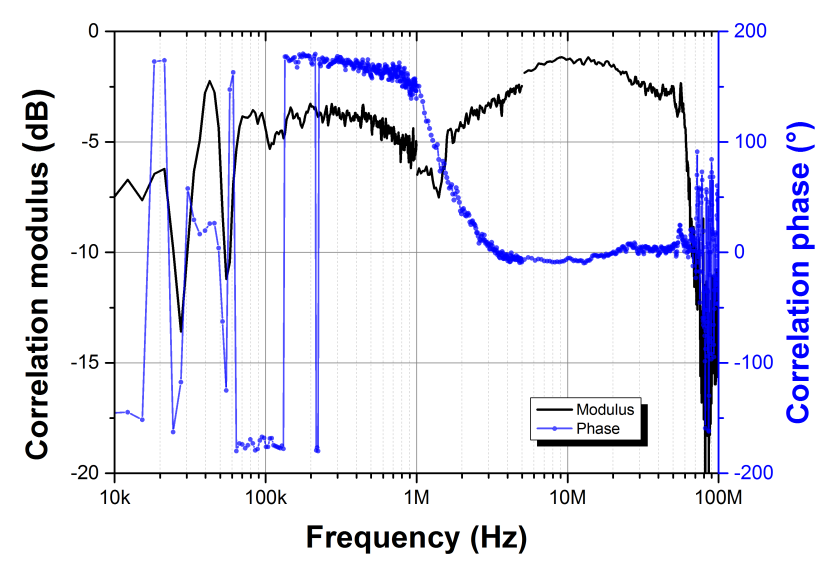

Figure 5: Intensity noise correlation function with modulus (black) and phase (blue).

\section{EVALUATION OF THE LASER FREQUENCY NOISE AND SPECTRAL LINEWIDTH}

The frequency noise of our laser source, as it affects light-atom interactions, is also evaluated in order to predict the CPT clock performance.

\subsection{Frequency noise}

The laser frequency noise $S_{v}(f)$, which reveals the fluctuations of the optical frequency, is measured in two different ways. First we compute the frequency noise with regard to a Cs hyperfine atomic transition using the error signal of the wavelength stabilization loop (Fig. 4). The frequency discriminator has a slope of $12 \mathrm{MHz} / \mathrm{V}$ on a linewidth corresponding to the hyperfine transition $(\sim 10 \mathrm{MHz})$. However it has a low bandwidth of $1.5 \mathrm{kHz}$ limited by the lock-in amplifier. Consequently, to measure the frequency noise above this cut-off frequency, the power spectrum density of the error signal needs to be corrected of the lock-in amplifier transfer function. Second, we use a Fabry-Perot interferometer (FPI, finesse 120 and free-spectral range $1.5 \mathrm{GHz}$ ) as a frequency discriminator. The signal is then much more sensitive to frequency fluctuations ( $3 \mathrm{MHz} / \mathrm{V})$ and has a bandwidth higher than $100 \mathrm{kHz}$. Both methods showed good agreements with each other and enable access simultaneously to the frequency fluctuations of the ordinary mode using the lock error signal to the Cs transition, and of the extraordinary mode measured by the FPI.

From these measurements, we conclude that the frequency noise of the two modes are similar to each other. Frequency noise power spectrum density (PSD) for the ordinary polarization is shown in figure 6 . It is mostly limited in the low frequency range by thermal and mechanical fluctuations (around $1 \mathrm{kHz}$ ) of the laser cavity. The contribution of the pump-induced thermal fluctuations inside the semiconductor chip - converted into a frequency noise through changes of the optical cavity length - has been experimentally measured at $6 \times 10^{5} \mathrm{~Hz}^{2} / \mathrm{Hz}$ on a bandwidth of $12 \mathrm{kHz}$ [12]. Because of the strong thermal resistance of our active structure $(\sim 50 \mathrm{~K} / \mathrm{W}$, with regard to incident pump power), it appears to be the main contribution to the noise frequency above $3 \mathrm{kHz}$. Under locked operation with feedback to the piezo-transducer, the frequency noise is suppressed on a bandwidth of $600 \mathrm{~Hz}$ limited by the piezo-transducer resonance. With both piezo and current feedback, the correction bandwidth is increased up to $10 \mathrm{kHz}$; in this case, the excess noise observed in figure 6 around $5 \mathrm{kHz}$ is balanced by a significant reduction of the frequency fluctuations at $1 \mathrm{kHz}$. The actual noise floor of these measurements results from the conversion of the laser $R I N$ into frequency noise through the servo-loop, and is estimated to $2.0 \times 10^{3} \mathrm{~Hz}^{2} / \mathrm{Hz}$. 


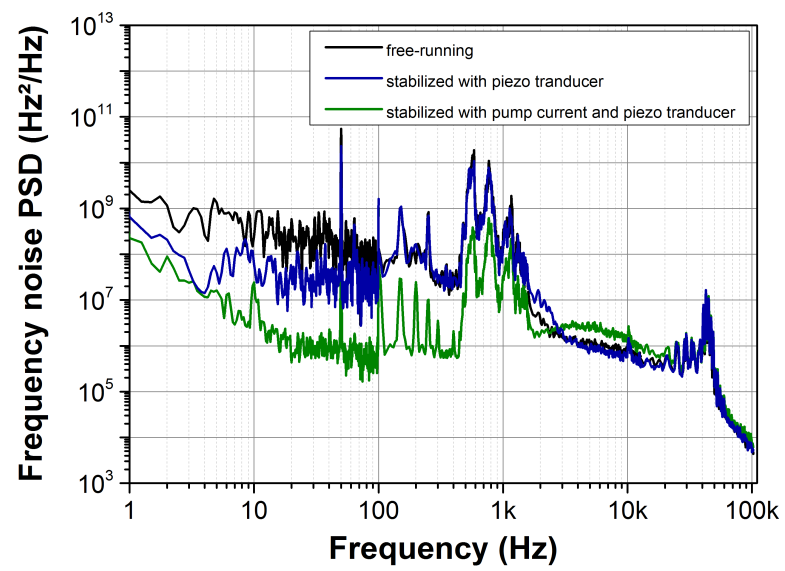

Figure 6: Experimental laser frequency noise of the ordinary polarized mode for different laser operation: in free running (black), in locked operation with piezo feedback (blue) and piezo / current feedback (green).

Finally we measured the correlation between the frequency fluctuations of the two modes following the protocol depicted previously for the intensity noise. For this experiment, the wavelength of the ordinary-polarized line was stabilized to the Cs transition with feedback to the piezo transducer glued to the output coupler. The signals used for correlation computation are the error signal from the wavelength lock for the ordinary mode, and the signal at side fringe of the FPI for the extraordinary polarized mode. Results are shown in figure 7. They show a strong correlation amplitude (modulus), higher than $-3 \mathrm{~dB}$, below $1 \mathrm{kHz}$. Above this frequency the correlation decreases down to the uncorrelated noise floor around $-20 \mathrm{~dB}$. The phase correlation could not be extracted from the data because of inversion of the frequency discriminator sign of the Fabry-Perot interferometer during the measurements. This result validates the benefit of a dual-frequency laser as compared to two separate lasers. In fact, as the two modes oscillate in the same cavity, they share the same mechanical and thermal fluctuations which ensures the phase coherence between them, without any active feedback loops.

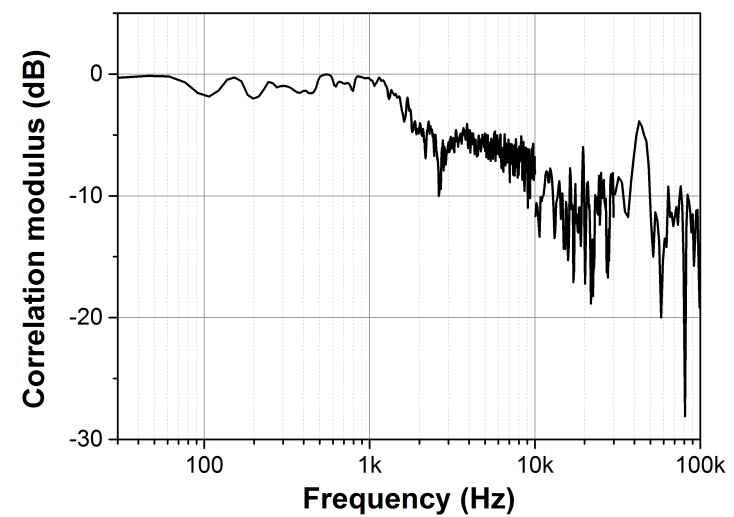

Figure 7: Experimental frequency correlation (modulus) between the two laser modes.

\subsection{Laser linewidth}

The laser linewidth is calculated by integrating the frequency noise spectral density, for different integration time $T_{\text {integration }}$, following the formula [13]

$$
L_{v}(v) \propto T F\left[\exp \left(-2 \int_{\frac{1}{T_{\text {Integration }}}}^{\text {fmax }} \frac{\sin ^{2}(\pi \tau f)}{f^{2}} S_{v}(f) d f\right)\right]
$$


where $f_{\text {max }}$ defines the maximum Fourier frequency on which the frequency noise is known, equal to $100 \mathrm{kHz}$ for our experiments. This limited upper frequency limits the validity of the numerical integration to $T_{\text {integration }} \geq 30 \mu \mathrm{s}$ integration time typically. Computations have been undertaken for the ordinary polarized mode and in different laser operations: under free-running, with feedback to the piezo transducer only and to both the piezo element and the pump driver current.

In addition, the evaluation of the laser spectrum has been completed by its actual measurement, in an experiment which provides the heterodyne beatnote signal between our dual frequency laser and an extended cavity diode laser (ECDL) used as a reference. The ECDL spectral line has been previously estimated [14], [15] and its linewidth is below $300 \mathrm{kHz}$ over 1 second integration time in our experimental conditions. The experimental set-up (Fig. 8) consists in superimposing the ECDL and dual-frequency ordinary beam, with the same polarization axis, on a fast photodiode to obtain a beatnote signal below $500 \mathrm{MHz}$. The signal is then amplified through two low-noise amplifiers with a $40 \mathrm{~dB}$ gain, and then measured with a RF spectrum analyzer (R\&S FSV-30). To avoid drifting of the ECDL wavelength which would results in the broadening of the beatnote, its wavelength is locked to a transition of Cs the hyperfine structure using a saturated absorption set-up.

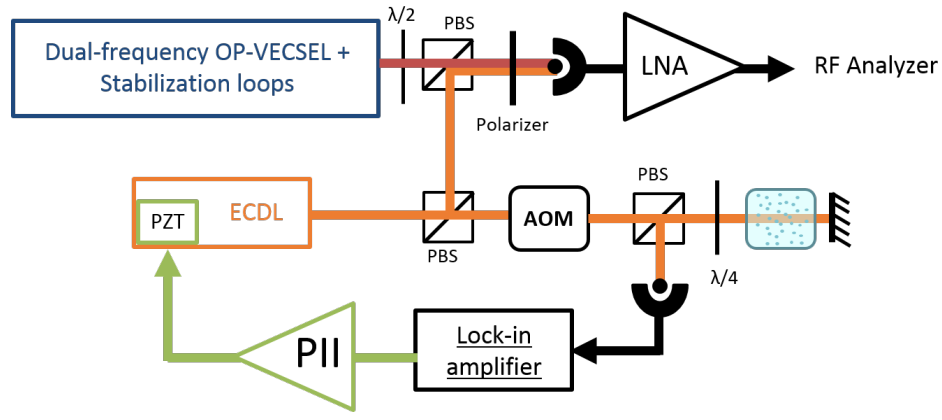

Fig 8: Experimental set-up for the heterodyne beatnote measurements. PBS: Polarizer Beam Splitter; $\lambda / 4$ : Quarter-wave plate; AOM: Acousto-optic modulator; PII: Proportional with 2 integration stages servo. LNA: Low-noise amplifier.

The spectrum linewidth displayed by the RF analyzer depends on its sweep time (associated to the width of the SPAN window). We thus defined an integration time for the beatnote measurements corresponding to the time taken by the RF analyzer to scan the full-width at half-maximum (FWHM) of the spectrum [16]. It is thus equal to:

$$
T_{\text {integration }}=\frac{F W H M}{S P A N} \times \text { Sweep Time }
$$

The beatnote spectrum has a Voigt profile which can be approximated by a Gaussian function close to the carrier frequency in order to extract the FWHM laser linewidth (Fig 9.).

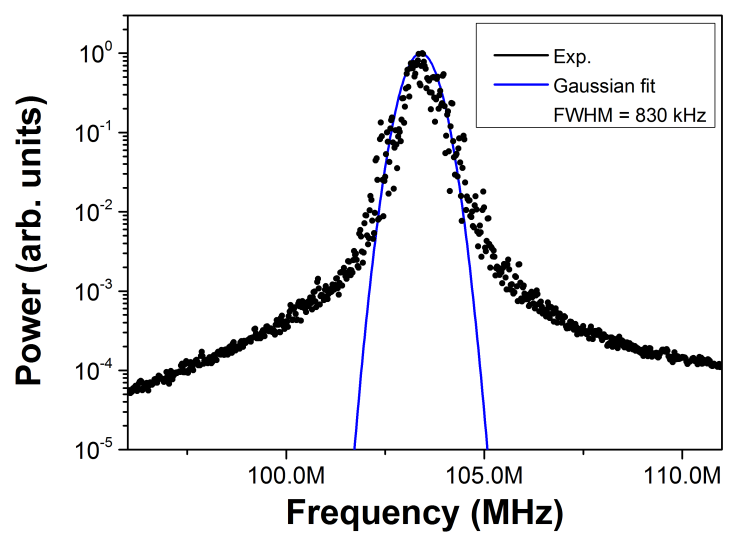

Figure 9: Experimental beatnote spectrum measure by the RF spectrum analyzer, and obtained between the ECDL, lock to 
Cs with feedback to piezo transducer, and the dual frequency laser ordinary mode, lock to Cs through feedback to both the piezo transducer and the pump driver current. The SPAN window is $25 \mathrm{MHz}$ and the sweep time is $0.195 \mathrm{~s}$. The FWHM of the Gaussian fit is $840 \mathrm{kHz}$ which gives an integration time of $11 \mathrm{~ms}$.

Experimental results of the laser linewidth for different integration times are shown in figure 10. Numerical computation of the frequency noise and heterodyne measurements are in good agreement with each other, demonstrating that in our experimental conditions the beatnote spectrum is limited by the dual-frequency laser linewidth. We observe a step-like evolution of the FWHM laser linewidth with the integration time, with a rapid decrease of the linewidth around $T_{\text {integration }}=1 \mathrm{~ms}$ associated to the main noise contribution around $1 \mathrm{kHz}$. The wavelength stabilization with correction on the piezo transducer reduces its long term-drift but is not able to reduce the laser linewidth on our measurement range. On the other hand, thanks to its $10 \mathrm{kHz}$ correction bandwidth, the current feedback loop reduces the laser linewidth to 1 $\mathrm{MHz}$ at $1 \mathrm{~s}$. However, measurements become very noisy for integration times between $10 \mu \mathrm{s}$ to $100 \mu \mathrm{s}$. In the worst case, we estimate the FWHM linewidth of our dual-frequency laser to $400 \mathrm{kHz}$ at $T_{\text {integration }}=100 \mu \mathrm{s}$.

As a conclusion, the linewidth of our laser is mainly limited by excess mechanical noises around $1 \mathrm{kHz}$, and electronic noises at $50 \mathrm{kHz}$. Without these contributions, the frequency noise would be limited by the pump-induced thermal fluctuations, which we should obtain by cancelling mechanical resonances in the laser cavity. In this case, numerical integration of the pump-induced frequency noise shows a linewidth below $500 \mathrm{kHz}$ at $1 \mathrm{~s}$. Additionally, it is worth noticing the RF beatnote generated by optical mixing of the two laser lines, in free running operation, exhibits a spectrum linewidth of $500 \mathrm{kHz}$ (FWHM) for a $300 \mathrm{~ms}$ integration time [8]. This value is below the actual laser linewidth of $3 \mathrm{MHz}$, confirming the strong correlation between the two laser frequencies as seen in figure 7

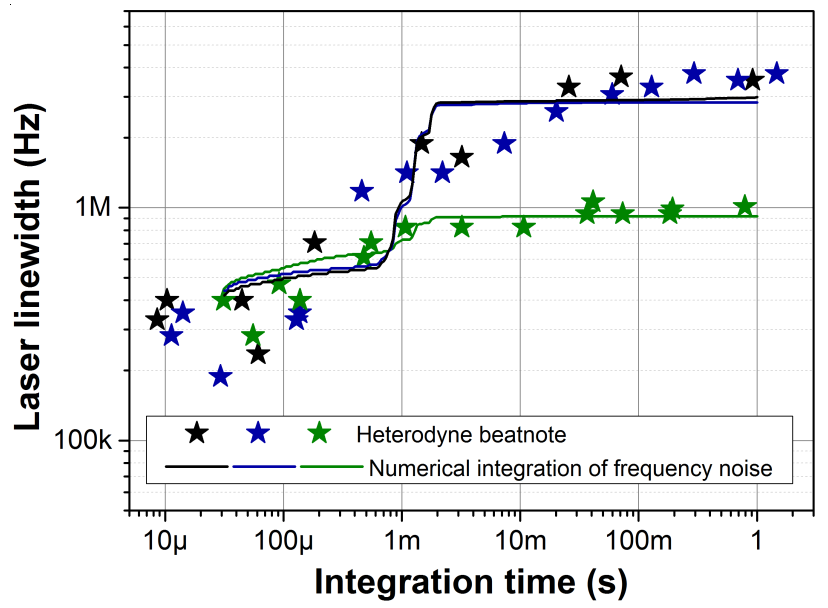

Fig 10: Evolution of the experimental laser linewidth for different integration time and for different laser operation: free running (black); lock with feedback to the piezo transducer only (blue); lock with feedback to the piezo transducer and the pump current feedback (green). Dots corresponds to heterodyne beatnote measurements and lines to numerical integration.

\section{CONCLUSION}

We have described the dual-frequency / dual-polarization emission of an optically-pumped semiconductor laser at 852 $\mathrm{nm}$, with a tunable frequency difference in the $\mathrm{GHz}$ range. It is based on the introduction of a controlled birefringence within the laser cavity, which results in two cross-polarized lasers lines. A compact prototype has been designed, which allows a fine tunability of the laser optical frequencies. The laser dual-frequency emission is stabilized by locking the ordinary wavelength to a Cs atomic transition in a saturated-absorption setup. The frequency difference is tunable in the $\mathrm{GHz}$ range. Under this working operation, the laser noise properties have been carefully investigated. Measurements show a maximum noise level of $-110 \mathrm{~dB} / \mathrm{Hz}$ for the laser relative intensity noise. In addition intensity correlation measurements reveals an anti-phase operation below $2 \mathrm{MHz}$. The laser frequency noise under locked operation is limited by excess mechanic and electronic noises resulting in a laser linewidth of $1 \mathrm{MHz}$ at $1 \mathrm{~s}$. Theoretical investigations show that with cancelation of this excess noise, the laser linewidth could be reduced to $500 \mathrm{kHz}$ at $1 \mathrm{~s}$, limited only by pump- 
induced frequency noise. The noise properties of our laser source are thus compatible with coherent population trapping experiments [8].

\section{ACKNOWLEDGMENTS}

Part of this work was funded by the French RENATECH network. The authors gratefully aknowledge support by Triangle de la Physique [2010-089T]; the Direction Générale de l'Armement; the Labex First-TF. The authors would like to thank Marc Hanna for helpful discussions about noise correlation measurements.

\section{REFERENCES}

[1] J. Vanier, “Atomic clocks based on coherent population trapping: a review," Appl. Phys. B, vol. 81, no. 4, pp. 421-442, Jul. 2005.

[2] S. Knappe, V. Shah, P. D. D. Schwindt, L. Hollberg, J. Kitching, L.-A. Liew, and J. Moreland, “A microfabricated atomic clock," Appl. Phys. Lett., vol. 85, no. 9, pp. 1460-1462, 2004.

[3] T. Zanon, S. Guerandel, E. de Clercq, D. Holleville, N. Dimarcq, and A. Clairon, "High contrast Ramsey fringes with coherent-population-trapping pulses in a double lambda atomic system.," Phys. Rev. Lett., vol. 94, no. 19, p. 193002, May 2005.

[4] J. Danet, M. Lours, and E. de Clercq, "Dick effect in a pulsed atomic clock using coherent population trapping," IEEE Trans. Ultrason. Ferroelect., Freq. Control., vol. 61, no. 4, pp. 567 - 574, 2014.

[5] G. Baili, L. Morvan, M. Alouini, D. Dolfi, F. Bretenaker, I. Sagnes, and A. Garnache, "Experimental demonstration of a tunable dual-frequency semiconductor laser free of relaxation oscillations.," Opt. Lett., vol. 34, no. 21, pp. 3421-3, Nov. 2009.

[6] F. A. Camargo, J. Barrientos, G. Baili, L. Morvan, D. Dolfi, D. Holleville, S. Guerandel, I. Sagnes, P. Georges, and G. Lucas-leclin, "Coherent Dual-Frequency Emission of a Vertical External-Cavity Semiconductor Laser at the Cesium D2 Line," IEEE Photonics Technol. Lett., vol. 24, no. 14, pp. 1218-1220, 2012.

[7] O. G. Okhotnikov, Semiconductor Disk Lasers: Physics and Technology. 2010.

[8] P. Dumont, F. Camargo, J. Danet, D. Holleville, S. Guerandel, G. Baili, L. Morvan, D. Dolfi, I. Gozhyk, G. Beaudoin, I. Sagnes, P. Georges, and G. Lucas-leclin, "Low-noise dual-frequency laser for compact Cs atomic clocks,” J. Light. Technol., vol. 32, no. 20, pp. 3817-3823, 2014.

[9] J. Danet, O. Koslova, P. Yun, S. Guérandel, and E. de Clercq, "Compact atomic clock prototype based on coherent population trapping," Eur. Phys. Journal, Web Conf., 2014.

[10] G. Baili, F. Bretenaker, M. Alouini, L. L. Morvan, D. Dolfi, and I. Sagnes, "Experimental Investigation and Analytical Modeling of Excess Intensity Noise in Semiconductor Class-A Lasers," J. Light. Technol., vol. 26, no. 8, pp. 952-961, 2008.

[11] S. De, V. Pal, a El Amili, G. Pillet, G. Baili, M. Alouini, I. Sagnes, R. Ghosh, and F. Bretenaker, "Intensity noise correlations in a two-frequency VECSEL.," Opt. Express, vol. 21, no. 3, pp. 2538-50, Feb. 2013.

[12] A. Laurain, M. Myara, G. Beaudoin, I. Sagnes, and A. Garnache, "Multiwatt-power highly-coherent compact single-frequency tunable vertical-external-cavity-surface-emitting-semiconductor-laser.," Opt. Express, vol. 18, no. 14, pp. 14627-36, Jul. 2010.

[13] K. Petermann, Laser Diode Modulation and Noise. Kluwer Academic Publishers, 1988.

[14] X. Baillard, A. Gauguet, S. Bize, P. Lemonde, P. Laurent, A. Clairon, and P. Rosenbusch, "Interference-filterstabilized external-cavity diode lasers," Opt. Commun., vol. 266, pp. 609-613, 2006.

[15] M. Gilowski, C. Schubert, M. Zaiser, W. Herr, T. Wu, T. Wendrich, T. Mu, E. M. Rasel, and W. Ertmer, "Narrow bandwidth interference filter-stabilized diode laser systems for the manipulation of neutral atoms," Opt. Commun., vol. 280, pp. 443-447, 2007.

[16] A. Laurain, "Source laser à semiconducteur à émission verticale de haute cohérence et de forte puissace dans le proche et le moyen infrarouge," Institut d'Electronique du Sud (IES) - Université Montpellier II, 2010. 